\title{
Margen positivo (R1) en la biopsia intraoperatoria durante una gastrectomía total por cáncer gástrico
}

\author{
Francisco Venturelli M. ${ }^{1,2}$
}

\section{Positive margin (R1) in the intraoperative biopsy during a total gastrectomy for gastric cancer}

Gastric cancer is a pathology with a high incidence in Chile, fortunately, the incidence of R1 margins is low when performing surgery with curative intent. When having a sub cardial or cardial tumor, it is essential to perform a rapid (intraoperative) biopsy, if this is positive when cutting the esophagus just under the pillars, it is difficult to make the decision of what to do next, since performing an inthrathoracic esophagojejunostomy increases the surgical risk. For this, it is very important to have information about the histopathological characteristics of the tumor, the leakage risk, there currence, the survival, the adjuvant therapies and the reality of the country. A review of the literature was done in order to guide the decisions. Key words: stomach neoplasms; total esophagogastrectomy; pancreatoduodenectomy; total gastrectomy; positive margin.

\section{Resumen}

El cáncer gástrico es una patología de alta incidencia en Chile. Afortunadamente es baja la incidencia de márgenes R1 cuando se realiza una cirugía con intención curativa. Al tener un tumor subcardial o cardial es perentorio realizar una biopsia rápida (intraoperatoria), en caso que ésta resulte positiva, es difícil tomar una decisión sobre qué realizar a continuación, ya que el realizar una esofagoyeyunoanastomosis intratorácica aumenta el riesgo quirúrgico. Para esto, es de suma importancia tener la información de las características histopatológicas del tumor, el riesgo de filtración, la recurrencia, la sobrevida, las terapias adyuvantes y la realidad país. Se realizó una revisión de la literatura con el fin de poder guiar la toma de decisiones.

Palabras clave: neoplasia gástrica; esofagogastrectomía total; pancreatoduodenectomía; gastrectomía total; margen positivo.

\section{Introducción}

Aún es controversial la extensión del margen que se debe obtener en una gastrectomía oncológica con el fin de mejorar la sobrevida y disminuir la recidiva local $^{1,2}$. De las resecciones gástricas, entre el 2,89\% y $20 \%$ presentan margen microscópico positivo en la biopsia diferida ${ }^{3,4}$. Sin embargo, la recurrencia sintomática sería solo en un $13 \%$ de los casos y el factor pronóstico más importante en estos pacientes es el compromiso nodal, por sobre el estado del margen de sección ${ }^{5,6}$.

Cuando hay duda sobre el compromiso del margen de sección al realizar una gastrectomía por cáncer gástrico, es perentorio realizar una biopsia rápida intraoperatoria con el fin de dejar al paciente R0. Esta puede ser tanto del margen proximal como distal.

Con el fin de reducir el número de pacientes $\mathrm{R} 1$, se ha intentado establecer la extensión ideal de margen macroscópico de sección y así poder lograr una cirugía curativa. Según la guía japonesa del $2017^{2}$, los T1 deben alcanzar un margen de $2 \mathrm{~cm}$, en pacientes T2 el margen proximal debe ser de $3 \mathrm{~cm}$, en T3-T4 el margen proximal debe ser de al menos $3 \mathrm{~cm}$ para tumores con un patrón de crecimiento expansivo (Tipo 1 y 2) y $5 \mathrm{~cm}$ para aquellos con un patrón de crecimiento infiltrativo (Tipo 3 y 4).
'Hospital Base Osorno.

Osorno, Chile.

¿Universidad Austral de Chile.

Recibido el 31 de diciembre de 2018, aceptado para publicación el 30 de junio de 2019

Correspondencia a: Dr. Francisco Venturelli fcoventurelli@gmail.com 
En los pacientes con invasión esofágica se debe realizar un estudio intraoperatorio del margen de sección, sin embargo, este presenta un $2 \%$ de falsos negativos, lo cual está dado principalmente por la presencia de focos tumorales en la submucosa ${ }^{7}$. Por otra parte el tener un margen positivo además de ser un factor de mal pronóstico, aumenta el riesgo de progresión tumoral local ${ }^{8}$.

Al encontrar un margen positivo proximal en el intraoperatorio, es controversial el hacer o no una esofagogastrectomía, así como también el hacer una pancreatoduodenectomía al presentar un margen distal positivo.

Al tener un paciente $\mathrm{R} 1$ en el posoperatorio las posibilidades son: quimioterapia (QMT), quimioradioterapia (QMRDT) o reoperarlo. Sin embargo, debido a que la gran mayoría de estos casos son estadios avanzados III y IV y gran parte de las recomendaciones se basan en estudios retrospectivos, es difícil saber qué decisión tomar ${ }^{8}$. Para ello es importante conocer las características histopatologías de estos tumores, el riesgo de filtración, la recurrencia, la sobrevida, terapias adyuvantes y la realidad del país.

\section{Características histopatológicas tumorales de los pacientes con margen $R 1$}

Al tener un paciente con margen microscópico positivo se debe tener en cuenta en qué contexto se encuentra, para saber qué decisión tomar. Por ejemplo: la edad del paciente, el performance status, el tiempo operatorio, las comorbilidades, la expectativa de vida del paciente, las características del centro hospitalario en el cual se está operando, la experiencia del cirujano y especialmente las características histopatológicas del tumor.

Según el estudio retrospectivo Chino de Liang $\mathrm{Y}^{8}$, los tumores con margen positivo R1, son tumores que tienen mayor compromiso linfonodal $(\mathrm{p}=0,005)$, mayor compromiso en profundidad de la pared gástrica $(\mathrm{p}=0,004)$, mayor estadio TNM $(\mathrm{p}=0,005)$, mayor tamaño $(\mathrm{p}=0,013)$, mayor índice de tumores con crecimiento infiltrativo (Bormann III y IV) $(\mathrm{p}=0,004)$ y una mayor incidencia de enfermedad extranodal $(\mathrm{p}<0,001)$.

Según el estudio norteamericano colaborativo de 7 centros de estados unidos de Postlewait $\mathrm{LM}^{9}$, los tumores con margen positivo son más frecuentemente tumores del proximales, del tipo difuso, mal diferenciado y con células en anillo de sello.

En una revisión sistemática de pacientes R1 en gastrectomía total y subtotal hubo 781 pacientes con margen positivo microscópico (R1). De ellos 30 casos (4\%) fueron T1, 98 (13\%) fueron T2, y 653
(83\%) fueron T3 o T4. En 671 pacientes se describió el compromiso linfonodal, de estos 543 (81\%) casos eran $\mathrm{N}+$, por lo tanto, la gran mayoría son $\mathrm{T} 3-\mathrm{T} 4 \mathrm{o}$ $\mathrm{N}+$, que son los principales factores de mal pronóstico en el cáncer gástrico ${ }^{5,10}$.

\section{Riesgo de filtración}

En un estudio colaborativo, que incluyó 7 centros de Estados Unidos, se identificaron 520 casos entre los años 2000 y 2012 con información de la biopsia rápida del margen proximal. De ellos, 453 pacientes tuvieron un margen negativo (87\%) y 67 (13\%) tuvieron margen positivo. En 54 (80,6\%) de los 67 casos se practicó una re-resección hacia proximal, logrando convertir a 48 de ellos a margen negativo. En los 453 casos que tuvieron margen negativo se encontraron 6 falsos negativos $(1,3 \%)$. El porcentaje de filtración en el grupo R0 fue $4 \%$ vs $15 \%$ en los R 1 y $18 \%$ en los R0 que previamente eran R1 ( $p<0,01)$. En el índice de complicaciones hubo diferencias estadísticamente significativas: $38 \%, 52 \%$ y $60 \%$ respectivamente $(\mathrm{p}=0,01)^{11}$. En complicaciones mayores Clavien III-V también hubo diferencias estadísticamente significativas $(\mathrm{p}=0,02)$.

\section{Recurrencia}

Una de las razones por lo cual es necesario alcanzar un margen negativo, es disminuir el riesgo de recidiva local. En 2 estudios se demostró que la recurrencia más frecuente en pacientes $\mathrm{R} 1$ no fue local si no que locorregional o a distancia. Stiekema ${ }^{12}$, en un estudio prospectivo holandés, incluyó 110 pacientes con seguimiento mínimo de 9 meses, de 30 pacientes $\mathrm{R} 1$, solo $2(6,7 \%)$ tuvieron recurrencia a nivel de la anastomosis.

En un estudio retrospectivo en el Seoul National University Hospital, registraron 1.536 pacientes entre el 2001 y 2011 en estadio III y IV según la clasificación TNM 2009. De estos, 35 (2,3\%) pacientes tuvieron margen positivo, $15(42,9 \%)$ margen proximal, $21(60 \%)$ margen distal y 1 paciente (4,8\%) ambos márgenes; de estos sólo un paciente tuvo recidiva locorregional $(4,8 \%)$, el cual además tuvo recidiva peritoenal y hematógena ${ }^{8}$.

Bickenbach $\mathrm{KA}^{5}$, en un estudio prospectivo en el Memorial Sloan-Kettering Cancer Center, New York, publicó 108 pacientes con margen positivo. De estos, $11(10,18 \%)$ pacientes fueron a re-resección del margen, encontrando que solo $3(27 \%)$ de ellos, finalmente, tenían enfermedad microscópica residual en el trozo resecado y, además, de los 108 pacientes R1 sólo 14 (13\%) tuvieron recurrencia sintomática. 


\section{Sobrevida}

Conocer la sobrevida de los pacientes con R1 es trascendental para decidir la conducta a tomar frente a un paciente con margen positivo en la biopsia rápida.

Con respecto a esto hay varios estudios, sin embargo, la gran mayoría son retrospectivos ${ }^{8,12-14}$.

¿Si según las característica tumorales son estadios avanzados, realmente influye el margen positivo en la sobrevida?

En el estudio de Bickenbach $\mathrm{KA}^{5}$, al realizar un análisis multivariado, se concluyó que un margen $\mathrm{R} 1$ solo es un factor pronóstico en los pacientes T1 y $\mathrm{T} 2$ y en pacientes con menor a 3 linfonodos $(+)$.

Gertler $\mathrm{R}^{13}$, en un estudio prospectivo alemán, evidencia que la sobrevida media específica por tumor de los pacientes R 0 es 56,3 meses $v s 18$ meses $(\mathrm{p}<0,01)$ en los R1. Al comparar R0 vs R1 en los N3 la sobrevida media es similar: 15 vs 12 meses $(\mathrm{p}=0,07)$ y al comparar los estadios IIIC tampoco se encontró diferencia en la sobrevida media: $15 \mathrm{vs}$ 13 meses $(\mathrm{p}=0,156)^{13}$. En el estudio de Cheng JD ${ }^{14}$ al comparar 50 pacientes R0 vs $72 \mathrm{R} 1$, no hubo diferencia en cuanto a las sobrevida media $(p=0,198)$. Solo hubo diferencia en la sobrevida al comparar pacientes con un ${ }_{\mathrm{p}} \mathrm{N} \leq 2$ (hasta 6 ganglios + ).

El estudio de Liang $\mathrm{Y}^{8}$, en el Tianjin Medical University Cancer Institute and Hospital, concluye que los pacientes con estadio IIIC R0 no tienen diferencia en la sobrevida con los R1. Paralelamente, un estudio colaborativo publicado por Squires $\mathrm{MH}^{11}$ concluye que al ampliar el margen de sección de positivo a negativo, no mejora la sobrevida $(\mathrm{p}=0,14)$ global ni tampoco la sobrevida libre de enfermedad $(p=0,49)$.

\section{Terapia adyuvante}

También es importante, al enfrentar el margen positivo en la biopsia rápida, saber si existe alguna terapia efectiva en el posoperatorio. En el estudio de Siekeman $\mathrm{J}^{4}$, se compararon 40 pacientes $\mathrm{R} 1$ con QMRDT adyuvante vs 369 sin QRDT evidenciando que los pacientes con el primer tratamiento presentaban una mejor sobrevida a 5 años $(p=0,003)$.

En el estudio chino de cohorte retrospectiva, Zhou M. ${ }^{15}$, comparó 33 pacientes R1 con QMRDT vs 81 pacientes con QMT con igual TNM si bien la sobrevida a 5 años es similar, al evaluar los pacientes $\mathrm{N} 0-2$, estos se beneficiarían de agregar la RDT.

Por otra parte la NCCN, publica que en los pacientes R1 sin terapia neoadyuvante se debe dar QMRDT y en los con QMT neoadyuvante administrar QMRDT o QMT adyuvante y considerar también la re-resección en pacientes con QMRDT o QMT preoperatoria ${ }^{16}$.

\section{Esofagogastrectomía en Chile}

En la situación que uno se encuentre con un margen positivo en la biopsia rápida del borde de sección proximal, una opción es una re-resección de ese margen, si este resulta positivo para neoplasia, una opción es realizar una esofagogastrectomía si se tiene el colon estudiado y limpio. En Chile, solo hay 3 series publicadas por grupos de alta casuística de cáncer gástrico a nivel nacional. Esto hace suponer que el número de casos en varios centros o son esporádicos o presentan malos resultados. En total las 3 series suman 36 esofagogastrectomías, solo 21 casos presentan clasificación TNM 2010, 16 son estadios IIIC y IV, con una morbilidad de $80 \%$ y una mortalidad de 5,8\%. Solo 16 de ellos fueron R0 y presentaron una sobrevida media de 20 meses y sobrevida a 5 años de $39 \%^{17-19}$ (Tabla 1).

\section{Margen duodenal R1}

Al encontrarse durante una gastrectomía con un dudoso margen duodenal, se debe realizar biopsia rápida. En caso de que resulte positiva se podría intentar una ampliación del margen realizando una resección duodenal suprapapilar. En caso de que ésta vuelva a salir con el margen positivo, habría que decidir si agregar o no una pancreatoduodenectomía. La pancreatoduodenectomía en estos casos presenta una morbilidad y número de fístula

Tabla 1. Literatura chilena publicada

\begin{tabular}{|c|c|c|c|c|c|c|c|c|c|}
\hline Autor & Año & Años de estudio & n & Diseño & Filtración & R0 & R1 & $\mathbf{R} 2$ & SV 5 años \\
\hline Abularach $^{17}$ & 2011 & $2008-2010$ & 5 & $\mathrm{R}$ & $1(20 \%)$ & 5 & 0 & 0 & NR \\
\hline Butte $^{18}$ & 2011 & 2004-2008 & 9 & $\mathrm{R}$ & $0(0 \%)$ & 9 & 0 & 0 & $0 \%$ \\
\hline
\end{tabular}

R: Retrospectivo, NR: no reportado, SV: sobrevida. 
pancreática mayor que los pacientes que se operan de una pancreatoduodenectomía por un tumor periampular. Habitualmente, en estos casos se trata de un cáncer avanzado con baja sobrevida a 5 años, pero es mejor que el tratamiento paliativo (34\% vs $5,6 \% ; p=0,0064)^{20}$. En una revisión sistemática de pancreatoduodenectomía en pacientes con cáncer gástrico Roberts $\mathrm{P}^{21}$, refiere que estaría indicada en: infiltración de cabeza de páncreas, infiltración de segunda porción duodenal, invasión de vía biliar distal o cuando presentan adenopatías peripancreáticas.

Saka $^{22}$ publica que la sobrevida a 5 años aumenta de un $13 \%$ a $29 \%$ al realizar una resección pancreática. En el estudio de Bickenbach $\mathrm{KA}^{5}$ de 36 pacientes con R1 duodenal no se documentó ninguna recidiva local.

El año 2019 se publicó una revisión sistemática que incluyó 13 artículos con un total de 69 pacientes describiendo una sobrevida media de 26 meses,

Figura 1. Algoritmo de toma de decisión intraoperatoria en caso de R1 proximal. con una sobrevida a 5 años de 39,3\% (de los 69, 12 no fueron T4), con un 59\% de morbilidad, $31 \%$ de fístula pancreática y un $1,4 \%$ de mortalidad quirúrgica $^{23}$.

\section{Conclusiones}

Son pocos los estudios que nos ayudan a tomar una conducta frente a un margen positivo durante la gastrectomía. Al estar con buen margen para realizar una re-resección es lo aconsejable. El problema es cuando al continuar resecando el esófago, se requiere introducirse al mediastino y hacer una anastomosis esofagoyeyunal alta con alta dificultad quirúrgica, no logrando una anastomosis segura.

Al parecer el margen positivo, no solo es un margen positivo por sí sólo, si no que traduce que se está frente a una enfermedad agresiva, que está en una etapa avanzada y que presenta una baja sobrevida independiente de la conducta que se tome.

Siempre al enfrentar una biopsia rápida de margen de sección positiva, se deben considerar múltiples factores:

1. La etapa de la enfermedad.

2. Las características del paciente.

3. La baja probabilidad de que recurra a nivel local

4. Que existe la posibilidad de que la biopsia rápida sea positiva y que el rodete esofágico del stapler resulte negativo.

5. Que el dejarlos R1 habitualmente no afecta mayormente la sobrevida, salvo en pacientes con estadios precoces (hasta la muscular y menor a 3 linfonodos positivos).

6. La morbimortalidad del centro quirúrgico.

7. Que la QMRT y QMT adyuvante han demostrado efectividad en estos casos.

8. Mientras más alta sea la anastomosis mayor es la posibilidad de filtración.

Los resultados de varias cirugías digestivas complejas como la esofagectomía y pancreatoduodenectomía dependen tanto de la experiencia del centro y del cirujano ${ }^{24}$. La sobrevida y mortalidad de la esofagogastrectomía por cáncer, en grandes centros de Chile, con cirujanos con experiencia sería aceptable. Sin embargo, en un centro de baja experiencia, tanto la esofagogastrectomía como pancreatoduodenectomía en cáncer gástrico, con el fin de dejar al paciente R0, no serían aconsejables debido a que probablemente estemos exponiendo al paciente a una cirugía donde los riesgos superan los beneficios (Figura 1). 


\section{Responsabilidades éticas}

Protección de personas y animales. Los autores declaran que para esta investigación no se han realizado experimentos en seres humanos ni en animales.
Confidencialidad de los datos. Los autores declaran que en este artículo no aparecen datos de pacientes.

Conflictos de interés: no hay.

\section{Bibliografía}

1. Japanese Gastric Cancer Association. Japanese Gastric Cancer Treatment Guidelines 2014 (ver. 4). Gastric Cancer. 2017;20:1-19.

2. Squires MH, Kooby DA, Poultsides GA, Pawlik TM, Weber SM, et al. Is it time to abandon the 5 -cm margin rule during resection of distal gastric adenocarcinoma? A multi-institutionstudy of the U.S. Gastric Cancer Collaborative. Ann Surg Oncol 2015;22:1243-51.

3. Morgagni P, La Barba G, Colciago E, Vittimberga G, Ercolani G. Resection line involvement after gastric cancer treatment: handle with care. Updates in Surgery 2018;70:213-23.

4. Stiekema J, Trip AK, Jansen EP, Aarts MJ, Boot $\mathrm{H}$, Cats A, et al. Does adjuvant chemoradio therapy improve the prognosis of gastric cancer after an $\mathrm{R} 1$ resection? Results from a dutch cohort study. Ann Surg Oncol. 2015;22:581-8.

5. Bickenbach KA, Gonen M, Strong V, Brennan MF, Coit DG. Association of positive transaction margins with gastric cancer survival and local recurrence. Ann Surg Oncol.2013;208:2663-8.

6. Yokota T, Kunii Y, Teshima S, Yamada Y, Saito T, Takahashi M, et al. Clinicopathologic prognostic features in patients with gastric cancer associated with esophageal or duodenal invasion. Ups J Med Sci 1999;104:217-30.

7. Woo JW, Ryu KW, Park JY, Eom BW, Kim MJ, Yoon HM, et al. Prognostic impact of microscopic tumor involved resection margin in advanced gastric cancer patients after gastric resection. World J Surg 2014;38:439-46.

8. Liang Y, Ding X, Wang X, Wang B, Deng J, Zhang L, et al Prognostic value of surgical margin status in gastric cancer patients. ANZ J Surg. 2014;85:678-84.
9. Postlewait LM, Maithel SK. The importance of surgical margins in gastric cancer. J Surg Oncol. 2016;113:277-82.

10. Aurello P, Magistri P, Nigri G, Petrucciani $\mathrm{N}$, Novi L, AntolinoL, et al. Surgical management of microscopic positive resection margin after gastrectomy for gastric cancer: a systematic review of gastric R1 management. Anticancer Res. 2014;34:6283-8.

11. Squires MH III, Kooby DA, Pawlik TM, Weber SM, Poultsides G, Schmidt $\mathrm{C}$, et al. Utility of the proximal margin frozen section for resection of gastric adenocarcinoma: A 7-Institution Study of the US Gastric Cancer Collaborative. Ann Surg Oncol. 2014;21:4202-10.

12. Stiekema J, Trip AK, Jansen EP, Boot H, Cats A, Ponz OB, et al. The prognostic significance of an R1 resection in gastric cancer patients treated with adjuvant chemoradiotherapy. Ann Surg Oncol. 2014;21:1107-14.

13. Gertler R, Richter J, Stecher L, Nitsche U, Feith M. What to do after R1-resection of adenocarcinomas of the esophagogastric junction? J Surg Oncol. 2016;114:428-33.

14. Chen JD, Yang XP, Shen JG, Hu WX, Yuan XM, Wang LB. Prognostic improvement of reexcision for positive resection margins in patients with advanced gastric cancer. Eur J Surg Oncol. 2013; 39:229-34.

15. Zhou M, Li G, Yang W, Deng W, $\mathrm{Hu}$ R, Wang Y, et al. Adjuvant chemoradiotherapy versus adjuvant chemotherapy for R1 resected gastric cancer: a retrospective cohort study. $\mathrm{Br} \mathrm{J}$ Radiol. 2018; 91: 20180276.

16. National Comprehensive Cancer Network (NCCN). NCCN Clinical Practice Guidelines in Oncology (NCCN guidelines), Gastric Cancer, version 3.2015. http://www.nccn.org/professionals/ physi cian_gls/pdf/gastric.pdf
17. Abularach CR, Venturelli MF, Cerda CR, Urizar A, Lira E, Haito Y, et al. Interposición de colon transverso como alternativa de reconstrucción tras la esofagogastrectomía total. Rev Chil Cir 2011;63:432-6

18. Butte JM, Waugh E, Parada H, De La Fuente H. Combined total gastrectomy, total esophagectomy, and D2 lymphnode dissection with transverse colonic interposition for adenocarcinoma of the gastroesophageal junction. Surg Today 2011;41:1319-23.

19. Ceroni M, Norero E, Henríquez, JP, Viñuela, E, Briceño, E, Martínez, C, et al. Total esophagogastrectomy plus extended lymphadenectomy with transverse colon interposition: A treatment for extensive esophagogastric junction cancer. World J Hepatology 2015;7:2411-7.

20. Wang XB, Yang LT, Zhang ZW, Guo JM, Cheng XD. Pancreaticoduodenectomy for advanced gastric cancer with pancreaticoduodenal region involvement. World J Gastroenterol. 2008;14:3425-9.

21. Roberts P, Seevaratnam R, Cardoso R, Law C, Helyer L, Coburn N. Systematic review of pancreaticoduodenectomy for locally advanced gastric cancer. Gastric Cancer 2012;15:S108-S15.

22. Saka M, Mudan SS, Katai H, Sano T, Sasako M, Maruyama K. Pancreaticoduodenectomy for advanced gastric cancer. Gastric Cancer. 2005;8:1-5.

23. Li DB, You J, Wang SJ, Zhou YM. Pancreaticoduodenectomy for locally advanced gastric cancer: Results from a pooled analysis. Asian J Surg. 2019;42:477-81.

24. Macedo F, Jayanthi P, Mowzoon M, Yakoub D, Dudeja V, Merchant N. The impact of surgeon volume on outcomes after pancreaticoduodenectomy: a meta-analysis. J Gastrointest Surg. 2017;21:1723-31 